\title{
El rol de las Cortes y la protección de la democracia: una aproximación desde regímenes transicionales ${ }^{* * *}$
}

\section{The role of the Courts sustaining democracy: An approach from transitional regimes}

SUMARIO

Introducción. I. Tribunales constitucionales, democracias transicionales y constitucionalismo abusivo. II. Rescatando la democracia: Colombia e India. 2.1. Colombia. 2.2. India. III. La derrota judicial: Venezuela y Hungría. 3.1. Venezuela. 3.2. Hungría. Conclusiones.

RESUMEN

Este artículo explora los factores institucionales y políticos que permiten o impiden la consolidación de un Tribunal Constitucional en democracias en transición como la colombiana y la venezolana. Estas democracias, a su turno, son susceptibles de ser víctimas de intentos de constitucionalismo abusivo por parte de ejecutivos poderosos, fenómeno típico de democracias jóvenes. Este escrito analiza casos constitucionales exitosos y fracasados de Europa Central, Asia y Latinoamérica para efectos de identificar cuáles

* Profesor de Derecho Constitucional de la Universidad de La Sabana (Colombia). Ha trabajado la legitimidad democrática del juez constitucional en el marco del proyecto de investigación "La Corte Constitucional y las reformas a la Constitución: de la incertidumbre a la razonabilidad", financiado por la Universidad de La Sabana. Autor de Constitución Popular, no Judicial, Bogotá, Temis, 2014.Contacto: vicente.benitez@unisabana.edu.co Agradezco los comentarios por parte de los profesores Richard Albert y YANIV RozNAi, a raíz de la presentación de este artículo en la Fifth Annual YCC Global Conference en la Universidad de Tulane (Estados Unidos).

** Abogado de la Universidad Javeriana, licenciado en Derecho de la Universidad del País Vasco con maestría en Derechos Fundamentales de la Universidad Carlos III de Madrid. Actualmente cursa una maestría en Derecho en la Universidad de Washington en St. Louis, Missouri, con especialización en Negociación y Resolución de Conflictos. Contacto: g.gonzalezherrera@wustl.edu

*** Recibido el 30 de enero de 2016, aprobado el 16 de mayo de 2016.

Para citar el artículo: V. F. Benítez R. y G. A. GonzÁLEz H., El rol de las Cortes y la protección de la democracia: una aproximación desde regímenes transiciomales, Derecho del Estado n. ${ }^{\circ}$ 36, Universidad Externado de Colombia, enero-junio de 2016, pp. 41-67. Dor: http://dx.doi. org/10.18601/01229893.n36.02. 
factores propician o frenan la posibilidad de un control de constitucionalidad efectivo en nuevas democracias. El artículo concluye que aspectos tales como la independencia judicial, la separación de los poderes, el prestigio de las Cortes y la deferencia judicial hacia otras ramas son cruciales para identificar si un Tribunal será exitoso.

PALABRAS CLAVE

Control jurisdiccional, democracia, revisión de la Constitución, Colombia, Venezuela, Hungría, India, derecho comparado, Corte Constitucional.

\section{ABSTRACT}

This article explores the political factors that enable or prevent the consolidation of an effective judicial review in transitional democracies such as the Colombian and the Venezuelan ones. These democracies, in turn, are likely to be victims of abusive constitutionalism attempts by powerful executives, which is a phenomenon typical of young democracies. This paper analyzes key cases of successful and unsuccessful experiences in Central Europe, Asia and Latin America in order to identify what kind of factors allow or hinder a Tribunal's effective judicial review in new democracies. I argue that factors as judicial independence, separation of powers, Courts' reputation, and judicial deference to other branches, are key elements to determine whether a Tribunal will be a successful institution .

\section{KEYWORDS}

Judicial review, democracy, constitutional revision, Colombia, Venezuela, Hungary, India, Comparative Law, Constitutional Court.

\section{INTRODUCCIÓN}

En el año 2009 el Tribunal Supremo venezolano consideró que un proyecto de enmienda (refrendado popularmente) a la Constitución de 1999 que permitía la reelección indefinida del Presidente era constitucional porque no se quebrantaban los principios de igualdad y alternancia en el poder ${ }^{1}$. Por su parte, en 2010 la Corte Constitucional colombiana consideró que un proyecto de reforma (con refrendación ciudadana) a la Constitución de 1991 que permitía una segunda reelección presidencial era inconstitucional 
puesto que se quebrantaban principios constitucionales como los de igualdad y alternancia en el poder ${ }^{2}$.

La pregunta que surge en este punto tiene que ver con cuáles son las razones que permiten que dos tribunales que operan bajo regímenes constitucionales similares (que se declaran democráticos) y con presidentes carismáticos tomen decisiones opuestas frente a los límites que debe observar el ejecutivo. La tesis que se defenderá es la siguiente: esta diversidad de decisiones no obedece a criterios netamente jurídicos (de interpretación-aplicación de normas) sino a razones políticas e institucionales referidas al rol que debe cumplir un Tribunal Constitucional en la consolidación de una democracia transicional ${ }^{3}$. Más específicamente, la tesis que se propondrá es que un factor crucial (entre otros) que facilita la existencia de un control de constitucionalidad fuerte está relacionado con el prestigio constitucional que la Corte despliega hacia la opinión pública y hacia otras ramas del poder. En tal sentido, si una Corte es más prestigiosa en términos constitucionales, le será más sencillo desarrollar un control estricto sobre el ejecutivo y el legislativo, de tal modo que esta podrá ser catalogada como un control verdaderamente operativo, dado que la Corte será considerada como la verdadera guardiana de la democracia. Por el contrario, la ausencia de una trayectoria y prestigio constitucionales dará lugar a que exista una mayor probabilidad de que la Corte sea debilitada y finalmente dominada por el ejecutivo por medio de sucesivas reformas constitucionales y legales que no pueden ser anuladas por la Corte, dado el inminente riesgo de que pueda ser eliminada por vías jurídicas.

Debe resaltarse que esta indagación adquiere aún más relevancia en nuevas democracias en las cuales cortes constitucionales recién creadas son más proclives a ser cooptadas o incluso abolidas por gobernantes poderosos, en comparación con democracias consolidadas o maduras como la norteamericana. En tal virtud, por ejemplo, son reveladores los casos de Venezuela y Colombia que están inmersos en una problemática constitucional global-que algunos autores han denominado como "constitucionalismo abusivo"- propia de algunas democracias constitucionales jóvenes ${ }^{4}$. Así las cosas, se trata de Estados con constituciones que incorporan estándares globalmente aceptados (derechos, democracia y tribunales constitucionales capaces de contener

2 Cfr. Corte Constitucional de Colombia, Sentencia C-141 de 2010. Fecha de consulta: 27 de mayo de 2015. Disponible en: http://www.corteconstitucional.gov.co/relatoria/2010/c-141-10. htm

3 Cfr. Moustafa, Tamir y Ginsburg, Tom. Introduction: The Function of Courts in Authoritarian Politics. En T. Ginsburg y T. Moustafa, eds., Rule by Law: The Politics of Courts in Authoritarian Regimes. Nueva York: Cambridge University Press, 2010, 3; Ginsburg, Tom. Judicial Review in New Democracies. Nueva York: Cambridge University Press, 2006, 11 y HIRSCHL, R. The New Constitutionalism and the Judicialization of Pure Politics Worldwide. Fordham Law Review, 2006, 75(2), 754.

4 Véase, por todos, Landau, D. Abusive Constitutionalism. U. C. Davis Law Review, $2013,47(1), 189-260$. 
decisiones supermayoritarias antidemocráticas, como ciertas enmiendas constitucionales) pero que, simultáneamente, cuentan con líderes (presidentes o primeros ministros) y partidos políticos muy populares dependientes de ellos, que usan las herramientas contenidas en la misma Constitución para fines antidemocráticos usualmente asociados con la eliminación de controles efectivos y con la perpetuación en el poder del gobernante y su partido ${ }^{5}$. Estas particularidades producen un ambiente en el que las restricciones que la Corte le impone al ejecutivo-legislativo podrían generar más fácilmente algunos contrataques.

En ese contexto, este artículo persigue dos cometidos: por un lado, evaluar los factores que han permitido que algunas nuevas cortes constitucionales hayan sido capaces de impedir la consolidación del poder ejecutivo, como ocurrió en el caso colombiano. Por otro lado, examinar los factores que han imposibilitado que otras cortes puedan ser consideradas como límites efectivos frente a los abusos constitucionales del ejecutivo, como en la experiencia venezolana. A su vez, estos factores no pueden ser explicados ni entendidos desde un punto de vista estrictamente jurídico y local. Por el contrario, dado que esta clase de asaltos constitucionales (i.e., por medio del uso de instrumentos jurídicos) es un fenómeno que generalmente se produce en nuevas democracias (especialmente en el Sur Global) que comparten valores constitucionales comunes, es útil comparar algunas experiencias alrededor del mundo desde una perspectiva política e institucional que podría complementar el análisis en derecho. En ese marco, este escrito, en primer lugar, explora las principales características del constitucionalismo abusivo y el rol político e institucional de las cortes constitucionales en democracias en transición. Posteriormente, y a la luz de estas consideraciones, se estudian dos experiencias constitucionales exitosas en las cuales las cortes han impedido la consolidación de un régimen constitucional autoritario (Colombia e India) y dos modelos constitucionales cuyas cortes han fracasado en dicha empresa (Venezuela y Hungría), con el fin de resaltar aquellos aspectos que han producido cortes fuertes y débiles, respectivamente. Finalmente se ofrecen algunas conclusiones.

\section{TRIBUNALES CONSTITUCIONALES, DEMOCRACIAS TRANSICIONALES Y CONSTITUCIONALISMO ABUSIVO}

Después del colapso de la Unión Soviética, el mundo, y especialmente el Sur Global (Latinoamérica, el Sureste Asiático, África y Europa Central y Oriental), vivió una ola de expedición de nuevas constituciones ${ }^{6}$. En efecto,

5 Cfr. Issacharoff, S. Constitutional Courts and Consolidated Power. The American Journal of Comparative Law, 2014, 62(3), 585.

6 Cfr. Gardbaum, S. Are Strong Constitutional Courts Always a Good Thing for New 
después del movimiento constitucional liberal de los siglos XVII y XVIII -que daría lugar a las constituciones de Estados Unidos y varios Estados europeos- y de las constituciones de la segunda posguerra -como el caso de las actuales constituciones de Alemania y España, entre otras-, un tercer movimiento constitucional irrumpió en el mundo. Los Estados de esta tercera ola del constitucionalismo global acogieron la idea según la cual era necesaria la presencia de una generosa carta de derechos, así como una serie de instituciones independientes del poder ejecutivo que tuviesen la posibilidad de controlarlo con el propósito de evitar el regreso a regímenes marcadamente autoritarios como los experimentados en ciertos Estados latinoamericanos por cuenta de las dictaduras militares o en Europa Oriental debido al férreo régimen comunista al cual estaban sometidos ${ }^{7}$.

Como consecuencia de esto, una de las instituciones que se ha expandido a lo largo de estos Estados que han transitado del autoritarismo a la democracia (o democracias en transición) es la del judicial review ${ }^{8}$. De acuerdo con ISSACHAROFF $^{9}$, uno de los paradigmas de estas nuevas democracias resulta ser la creación de tribunales cuya función primordial es evitar el regreso a un régimen autocrático gracias a un control fuerte de las decisiones mayoritarias emanadas del ejecutivo o del legislativo. En tal sentido, una de las misiones principales de estas instituciones judiciales empoderadas por estas constituciones era mantener un régimen democrático que privilegiase la rotación de los partidos políticos (competencia electoral) y protegiese los derechos de las minorías o, lo que es lo mismo, cortes que previniesen la consolidación de un régimen con un partido único o un líder dominante ${ }^{10}$. Para lograr tal objetivo, estos tribunales tendrían la posibilidad de anular e intervenir en las medidas expedidas por el parlamento o por el ejecutivo y, de este modo, mejorar la democracia mediante decisiones judiciales promotoras de la deliberación democrática, de los derechos y de la competencia electoral ${ }^{11}$.

No obstante, este rol de los jueces en democracias jóvenes ha ocasionado que estos sean percibidos por el gobierno como actores políticos cuyas decisiones -judiciales en principio- intervienen indebidamente en asuntos de alta política, es decir, actores que han judicializado la megapolítica ${ }^{12}$. Así, esta nueva actitud judicial en estas democracias transicionales encontraría la

Democracies? Columbia Journal of Transnational Law, 2014-2015, 53(2), 287 e Issacharoff, S. Constitutional Courts and Democratic Hedging. Georgetown Law Journal, 2010-2011, 99(4), 965.

7 Véase GinsBURG, ob. cit., 90.

8 Cfr. Gardbaum, ob. cit., 291; Ginsburg, ob. cit., 6 e IssacharofF, S. Constitutionalizing Democracy in Fractured Societies. Texas Law Review, 2003-2004, 82(7), 1862-1853.

9 Véase Issacharoff, Constitutional Courts and Consolidated Power, cit., 585.

10 Cfr. ibíd., 589.

11 Cfr. ibíd., 589, e Issacharoff, Constitutional Courts and Democratic Hedging, cit., 965.

12 Cfr. ibíd., 964, y HiRSCHL, ob. cit., 721-728. 
resistencia de aquellos partidos y líderes carismáticos que consideran que estas decisiones judiciales-políticas son obstáculos que deben ser removidos puesto que afectan valores centrales de su política gubernamental y son contramayoritarias ${ }^{13}$. En tal sentido, estos líderes y/o partidos se enfrentan al siguiente dilema: por un lado, pueden cumplir con estas decisiones judiciales adversas (con lo cual su programa de gobierno se truncaría); por el otro, pueden tratar de eludir su cumplimiento (con lo cual su credibilidad constitucional ante el mundo se afectaría seriamente). Ante tal situación, la forma de resolución de esta encrucijada por parte de algunos de los gobiernos ha sido el recurso a lo que LANDAU ${ }^{14}$ y otro ${ }^{15}$ han denominado como "constitucionalismo abusivo", esto es, "el uso de mecanismos de cambio constitucional con el fin de hacer un Estado significativamente menos democrático de lo que era antes" ${ }^{16}$. Asî las cosas, usando instrumentos constitucionales, vale decir, cumpliendo con el derecho vigente, los gobiernos evitan que las cortes interfieran en sus decisiones políticas ${ }^{17}$ gracias a diversas estrategias como las siguientes:

- Aprobación de reformas constitucionales dirigidas a dejar sin efectos decisiones jurisprudenciales o a excluir de la jurisdicción de dichas cortes ciertos asuntos de interés gubernamental ${ }^{18}$.

- Implementación de planes de recomposición de los integrantes de la Corte para elegir jueces leales al gobierno ${ }^{19}$.

- Aprobación de nuevas constituciones (siguiendo los requisitos procesales) en las cuales estos tribunales tienen un papel político menos protagónico debido a una disminución sensible de sus funciones ${ }^{20}$.

Como advierte $\mathrm{VAROL}^{21}$, se trataría de una labor en la que, gracias al uso de mecanismos previstos constitucionalmente, se camuflan propósitos autoritarios. Así, aunque en apariencia el régimen político del Estado pareciese ser una democracia constitucional, en el fondo existe el dominio de un partido y/o un líder popular debido a la ausencia real de mecanismos de contrapeso. En suma, como señalan analistas de regímenes constitucionales abusivos, estos suponen la creación de un nuevo tipo de Estado

13 Issacharoff, Constitutional Courts and Consolidated Power, cit., 590 y 600.

14 LANDAU, ob. cit., 195.

15 Cfr. VArol, O. Stealth Authoritarianism. Iowa Law Review, 2015, 100 (4), 1674-1675, y Tushnet, M. Authoritarian Constitutionalism. Cornell Law Review, 2014-2015, 100(2), 433.

16 Traducción libre de LANDAU, ob. cit., 195.

17 Cfr. VAROL, ob. cit., 1678-1679 y 1685.

18 Hirschl, ob. cit., 758; Ginsburg, ob. cit., 77; LANDAu, ob. cit., 230, y Dixon, R. y LANDAU, D. Transnational constitutionalism and a limited doctrine of unconstitutional constitutional amendments. International Journal of Constitutional Law, 2015, 13(3), 613.

19 Cfr. Landau, D. A Dynamic Theory of Judicial Role. Boston College Law Review, 2014, 55(5), 1507; V AROL, ob. cit., 1688 y HirSCHL, ob. cit.

20 Cfr. Landau, Abusive Constitutionalism..., cit., 243.

21 Ob. cit., 1684. 
-híbrido ${ }^{22}$ - que se encuentra a medio camino entre Estados autoritarios tradicionales -irrespetuosos del Estado de derecho y sin competencia electoral-y regímenes constitucionales liberales -que son respetuosos del derecho y en los cuales existe competencia electoral ${ }^{23}-$. Por lo mismo, estos autores observan que este "camuflaje" constitucional para alcanzar cometidos antidemocráticos resulta ser mucho más difícil de combatir puesto que los criterios tradicionales (o check lists) usados para identificar si un Estado es autoritario son eludidos por estas nuevas formas de autoritarismo mimetizado y sutil, dado que ningún Estado está dispuesto a asumir los costos de imagen que implica aceptar explícitamente que ha asumido un orden antidemocrático ${ }^{24}$.

En ese contexto es posible decir que la actitud asumida por las cortes del Sur Global que desarrollan judicial review sobre enmiendas abusivas ha sido doble. Por una parte, se encuentran algunas experiencias exitosas de tribunales que se han enfrentado al gobierno y han anulado reformas constitucionales promovidas por este para (a) restringir los derechos de las minorías, (b) limitar las atribuciones del Tribunal encargado del judicial review o (c) consolidar el poder de un partido o gobernante mediante la eliminación o fuerte restricción de la competencia electoral ${ }^{25}$. Por otro lado, existen casos fallidos de intervenciones judiciales en los cuales las cortes han sido incapaces de detener el afianzamiento, por medio de enmiendas autoritarias, de regímenes constitucionales abusivos. Estas dos tendencias opuestas han impactado, a su vez, dos Estados suramericanos relativamente similares en términos constitucionales pero con cortes políticamente divergentes: Colombia y Venezuela. En ese sentido, a continuación se describen estos dos casos en conjunto con la experiencia exitosa de India y el caso fallido de Hungría, dado que, como ya se mencionó, el fenómeno del constitucionalismo abusivo y los intentos de las cortes por contenerlo no son exclusivamente latinoamericanos, sino globales $^{26} \mathrm{y}$, en consecuencia, los factores políticos e institucionales que facilitan o retrasan la acción de los jueces pueden ser discernidos más nítidamente si se considera que este es un problema general que sufren varias democracias surgidas en las postrimerías del siglo $\mathrm{xx}$, independientemente de su ubicación geográfica.

22 Cfr. Tushnet, ob. cit., 394; Moustafa y Ginsburg, ob. cit., 2 y Landau, Abusive constitutionalism..., cit., 189 y 199.

23 Cfr. VAROL, ob. cit.,1683.

24 Cfr. ibíd., 1676 y 1681.

25 Véase YAP, P. J. The Conundrum of Unconstitutional Constitutional Amendments. Global Constitutionalism, 2015, 4(1), 121 y 128; Bernal-Pulido, C. Transitional Justice within the Framework of a Permanent Constitution: The Case Study of the Legal Framework for Peace in Colombia. Cambridge Journal of International and Comparative Law, 2014, 3(4), 1141 y RoznAI, Y. Unconstitutional Constitutional Amendments - The Migration and Success of a Constitutional Idea. American Journal of Comparative Law, 2013, 61(3), 718.

26 Cfr. Albert, R. Amending Constitutional Amendment Rules. International Journal of Constitutional Law. 2015, 13(3), 658-659 y GARDBAUM, ob. cit., 287-288. 
II. RESCATANDO LA DEMOCRACIA: COLOMBIA E INDIA

En estos Estados, que a primera vista podrían parecer disímiles, existe un común denominador: un Tribunal, por medio de sus pronunciamientos, pudo desarticular eficazmente los intentos jurídicos de sus carismáticos líderes de afirmar su poder como gobernantes.

\subsection{Colombia}

En julio de 1991 Colombia expidió su actual Constitución que derogaría la centenaria Carta de 1886. En medio de un escenario de violencia, aupada por el narcoterrorismo que afectaba las grandes ciudades, varios sectores de la sociedad impulsaron un cambio constitucional que pudiese hacer frente a estos retos ${ }^{27}$. Sin embargo, la Constitución de 1886 podía ser modificada, según su artículo 218, solo si dicha enmienda era aprobada por el Congreso de la República. Esta institución se negó a adelantar estos cambios y, como consecuencia de ello, diversos movimientos sociales (liderados por el movimiento estudiantil de la "séptima papeleta") propiciaron un pronunciamiento popular en las urnas que dio lugar a la nueva Constitución ${ }^{28}$. Luego de algunos meses, una asamblea nacional constituyente redactó una Constitución que, por primera vez en la historia de Colombia, no resultaba ser la imposición de un partido político ${ }^{29}$, sino el consenso alcanzado por las tres principales fuerzas políticas del país en ese entonces ${ }^{30}$. Para evitar que la situación de bloqueo se repitiese, la Constitución estableció que su reforma podía ser adelantada no solo por el Congreso sino también por la ciudadanía ${ }^{31}$.

Una de las características más sobresalientes de la Constitución fue la creación de una Corte Constitucional poderosa, que tiene la capacidad de anular leyes o reformas constitucionales cuando estas sean inconstituciona$\operatorname{les}^{32}$. Durante los primeros años de su existencia, la Corte expidió sentencias garantistas de los derechos fundamentales en muy diversos campos,

27 Véase Dugas, J. The Origin, Impact and Demise of the 1989-1990 Colombian Student Movement: Insights from Social Movement Theory. Journal of Latin American Studies, 2011, 33(4), 808 y Quinche, M. F. Derecho Constitucional colombiano. De la Carta de 1991 y sus reformas. Bogotá: Temis, 2012, 17-23.

28 Cfr. CARrillo, F. Constitucionalizar la democracia social. El proceso constituyente de 1991 y América Latina. Bogotá: Temis, 2011, XVII, XVIII y 3-6.

29 En este sentido véase, entre otros, CEPEDA-Espinosa, M. J. Judicial Activism in a Violent Context: The Origin, Role, and Impact of the Colombian Constitutional Court. Washington University Global Studies Law Review, 2004, 3(4), 545 y VAlenCiA-VILla, H. Cartas de batalla. Una crítica del constitucionalismo colombiano. Bogotá: Panamericana, 2010.

30 Cfr. Segura, R. y Bejarano, A. ¡Ni una asamblea más sin nosotros! Exclusion, Inclusion, and the Politics of Constitution-Making in the Andes. Constellations, 2004, 11(2), 220.

31 Cfr. Uprimny, R. The Recent Transformation of Constitutional Law in Latin America: Trends and Challenges. Texas Law Review, 2011, 89(7), 1594.

32 Cfr. Cepeda-Espinosa, ob. cit., 557-558, así como Corte Constitucional de Colombia, 
especialmente en relación con las libertades individuales ${ }^{33}$ y los derechos sociales $^{34}$. De este modo, muchas personas vieron que sus reclamos podían ser eficazmente tramitados en la Corte como lugar donde vive la democracia en lugar del Congreso ${ }^{35}$. Igualmente, limitó de una manera estricta los estados de excepción que, bajo la anterior Constitución, permitieron la legislación permanente y casi incontrolada por parte del Presidente ${ }^{36}$. Finalmente, la Constitución prohibió la reelección presidencial como límite adicional al ejecutivo.

En ese panorama, en el año 2002 Álvaro Uribe fue elegido como Presidente de la República. Se trata de un líder carismático que llegó al poder con un amplio respaldo popular y por fuera de los partidos tradicionales. Ante el fracaso en los diálogos de paz con la guerrilla de las FARC que sufrió el anterior gobierno, URIBE prometió combatir a este grupo armado militarmente para recobrar el orden público que se había deteriorado a lo largo de los años $^{37}$. Para lograr ese objetivo, promovió varias reformas constitucionales que, para algunos, resultaban ser una instrumentalización de la democracia para consolidar su poder ${ }^{38}$. Algunas de ellas estaban destinadas a combatir el crimen mediante la restricción fuerte de derechos fundamentales y otras, dada la popularidad del presidente, buscaban modificar con la aquiescencia popular varias instituciones de otras ramas del poder. Sin embargo, una de las primeras reformas constitucionales más profundas fue permitir la reelección

Sentencia C-574 de 2011. Fecha de consulta: 27 de mayo de 2015. Disponible en: http://www. corteconstitucional.gov.co/relatoria/2011/c-574-11.htm

33 Cepeda-Espinosa, ob. cit., 575-626 y UPRIMnY, ob. cit., 1591.

34 Cepeda-Espinosa, ob. cit., 616-620 y Cepeda-Espinosa, M. J., Transcript: Social and Economic Rights and the Colombian Constitutional Court. Texas Law Review, 2010-2011, 89(7).

35 Cfr. Landau, A Dynamic Theory..., cit., 1514.

36 Cfr. CePEDA-Espinosa, Judicial Activism in a Violent..., cit., 631-635.

37 Issacharoff, Constitutional Courts and Consolidated..., cit., 590.

38 Muy diversos autores califican a Colombia como un caso típico de "constitucionalismo abusivo", en gran medida debido a la múltiples reformas constitucionales promovidas con la llegada del presidente Álvaro Uribe. Véase Albert, ob. cit.; YAP, ob. cit. y LANDAU, Abusive Constitutionalism..., cit. En el mismo sentido, algunos autores nacionales han calificado las diversas propuestas de reforma constitucional promovidas por el gobierno de ese entonces como una instrumentalización de la democracia. Cfr. OsunA, N. La sentencia del referendo: guarda de la Constitución ante el uso instrumental de la democracia. En Montealegre, E. coord., Anuario de Derecho Constitucional: análisis de jurisprudencia de la Corte Constitucional, período 2002 y primer semestre 2003. Bogotá: Universidad Externado de Colombia,24-27. Es interesante notar que varios autores coinciden en afirmar que la "política constitucional" del ex presidente Uribe fue la que desencadenó una nueva tesis por parte de la Corte Constitucional en el sentido de revisar las enmiendas constitucionales por vicios de competencia, además de por los tradicionales vicios de procedimiento. Cfr. CAJAS, M. A. El control judicial a la reforma constitucional, Colombia, 1910-2007. Cali: ICESI, 2008, 101-103 y RAMírez Cleves, G. El control material de las reformas constitucionales mediante acto legislativo a partir de la jurisprudencia establecida en la Sentencia C-551 de 2003. Revista Derecho del Estado. 2006, 18, 3. 
presidencial inmediata en Colombia por primera vez en más de 50 años ${ }^{39}$. De una manera ágil el Congreso (en donde el gobierno contaba con amplias mayorías) aprobó dicha enmienda. Miembros de la oposición veían con recelo esta reforma y la demandaron ante la Corte. A pesar de ello, esta declaró que una reelección presidencial por una única vez no alteraba principios esenciales de la Constitución porque las funciones del presidente no se incrementaban necesariamente con la extensión de su mandato ${ }^{40}$. A la postre, URIBE sería reelegido con un amplio respaldo popular para el periodo 2006-2010.

Nuevamente, en el año 2009 el Congreso aprobó una ley de referendo para una segunda reelección auspiciada por la coalición mayoritaria. No obstante, dicha reforma fue declarada inconstitucional ${ }^{41}$ porque la primera reelección había creado un desbalance con otros órganos de control, como ocurrió con la misma Corte Constitucional ${ }^{42}$, la Defensoría del Pueblo ${ }^{43}$ y el Banco de la República ${ }^{44}$, entre otros. Uribe acató la sentencia dado que, tradicionalmente, la Corte ha gozado de un gran respeto y los ejecutivos han cumplido con sus decisiones ${ }^{45}$. Como señala LANDAU ${ }^{46}$, la Corte evitó una erosión grave del régimen democrático colombiano y, posiblemente por eso, en 2015 se reformó la Constitución para prohibir la reelección presidencial ${ }^{47}$.

\subsection{India}

Tres años después de su independencia se expidió la Constitución de India de 1950. Uno de sus objetivos centrales era lograr la disminución de la desigualdad social ${ }^{48}$. Por esa razón, uno de los héroes de la independencia y su primer Jefe de Gobierno, JaWAHARLAL NEHRU, decidió promover una serie de

39 Véase Uprimny, ob. cit., 1598.

40 Cfr. Corte Constitucional de Colombia, Sentencia C-1040 de 2005. Fecha de consulta: 27 de mayo de 2015. Disponible en: http://www.corteconstitucional.gov.co/relatoria/2005/C-1040-05. htm

41 Véase Corte Constitucional de Colombia, Sentencia C-141 de 2010. Fecha de consulta: 27 de mayo de 2015. Disponible en: http://www.corteconstitucional.gov.co/relatoria/2010/c-141-10. htm

42 Véase Rubiano, S. La Corte Constitucional: entre la independencia judicial y la captura política. En M. García y J. Revelo, eds., Mayorías sin Democracia. Desequilibrio de poderes y Estado de Derecho en Colombia, 2002-2009. Bogotá: DeJusticia, 2009, 84-145.

43 Sobre el particular véase Revelo, J. La Defensoría del Pueblo: el silencio complaciente. En M. García y J. Revelo, eds., Mayorías sin Democracia, cit., 146-175.

44 Véase Rubiano, S. La independencia del Banco de la República y la reelección presidencial. En M. García y J. Revelo, eds., Mayorías sin Democracia, cit., 207-243.

45 Cfr. Posada-CARbó, E. Colombia after Uribe. Journal of Democracy, 2011, 22(1), 137-138.

46 Landau, Abusive Constitutionalism..., cit., 203.

47 Véase el Acto Legislativo 2 de 2015, art. 9. .

48 Cfr. Bhanu-Menta, P. The Rise of Judicial Sovereignty. Journal of Democracy, 2007, 18(2), 73 y Robinson, N. Expanding Judiciaries: India and the Rise of the Good Governance Court, Washington University Global Studies Law Review, 8(1), 2009, 4. 
leyes para redistribuir la propiedad. No obstante, diversas cortes estatales así como la Corte Suprema de Justicia anularon varias de esas medidas por considerar que con ellas se afectaba el derecho a la propiedad e igualdad de los propietarios ${ }^{49}$. Ante esta situación NeHRU, que contaba con una amplísima mayoría en el Parlamento ${ }^{50}$, aprobó la enmienda primera que excluía de la jurisdicción de la Corte Suprema el examen de cualquier intento de expropiación con fines redistributivos. La Corte, dada la ascendencia y liderazgo de NeHRU, en 1951 dio vía libre a esta disposición y señaló que no podía controlar la constitucionalidad de las enmiendas porque era una cuestión política ${ }^{51}$. De las primeras 45 enmiendas, la mitad estaban dirigidas a limitar a la Corte ${ }^{52}$.

Luego de la muerte de NeHRU, nuevas enmiendas se aprobaron para restringir la labor de la Corte. Sin embargo, en 1967 esa institución judicial conoció una de esas reformas que recortaban su competencia en relación con medidas expropiatorias y manifestó que: (i) las enmiendas en India se aprueban por medio de un tipo especial de ley; (ii) las leyes deben ser compatibles con los derechos fundamentales (art. 13.2 constitucional), y, por lo tanto, (iii) las enmiendas deben respetar los derechos fundamentales ${ }^{53}$. A pesar de que esta nueva tesis no se aplicó a la enmienda que se estaba examinando en ese momento, por razones de seguridad jurídica y para evitar un choque con el Parlamento, la carismática Primer Ministro IndiRa GandHI (hija de NeHru) configuró su campaña reeleccionista bajo la idea de que ese caso era una afrenta contra el poder del Parlamento y del Primer Ministro, y prometió reversar esta tesis jurisprudencial tan pronto como fuera reelegida ${ }^{54}$. En 1971 INDIRA GANDHI triunfaría con una mayoría aplastante en el Parlamento ${ }^{55}$, el cual aprobó entonces varias enmiendas promovidas por aquella que, por

49 Sobre el particular, véase a SATHE S. P. India: From Positivism to Structuralism. En J. Goldsworthy, ed., Interpreting Constitutions. A Comparative Study. Oxford: Oxford University Press, 2009, 239.

50 Véase Rajeev-Gowda, M. V. y Sridharan, E. Parties and the Party System, 19472006. En S. Ganguly et al., eds., The State of India's Democracy. Baltimore: The John Hopkins University Press, 2007, 8-9.

51 Sobre el particular, véase el fallo Sankari Prasad v. Union of India. Corte Suprema de Justicia de India, 1951. Fecha de consulta: 14 de enero de 2014. Disponible en: http://judis.nic. in/supremecourt/imgs 1 .aspx?filename $=1174$

52 Cfr. Bhanu-Mehta, ob. cit., 74.

53 Véase el caso Golaknath v. State of Punjab. Corte Suprema de Justicia de la India, 1967. Fecha de consulta: 27 de mayo de 2015. Disponible en: http://judis.nic.in/supremecourt/ imgs 1 .aspx ?filename $=2449$

54 Neuborne, B. The Supreme Court of India. International Journal of Constitutional Law, 2003, 1(3), 489 y SATHE S. P. Judicial activism in India. Transgressing Borders and Enforcing Limits. Nueva Delhi: Oxford University Press, 2011, 68.

$55 \mathrm{Al}$ respecto véase Das, G. The Supreme Court: An Overview. En B. N. KIRPAI et al., eds., Supreme but not Infallible, Essays in Honour of the Supreme Court of India. Nueva Delhi: Oxford University Press, 2011,21 y Austin, G. The Supreme Court and the Struggle for Custody of the Constitution. En KirPaI et al., eds., Supreme but not Infallible, cit., 4. 
una parte, eliminaban cualquier judicial review de las enmiendas y, por la otra, ordenaban nuevas expropiaciones. La crisis se profundizó cuando la Corte decidió dos casos que frenaron las expropiaciones de algunos bancos y unas tierras. En medio de fuertes presiones, la Corte expidió el que para muchos es el fallo más importante de su historia (Kesavananda Bharati v. State of Kerala $^{56}$ ) y en el cual concluyó que la Constitución de India tiene una estructura básica que le da su identidad y que no puede ser eliminada ni siquiera por una enmienda aprobada por una supermayoría ${ }^{57}$.

Ante esta nueva afrenta, Gandhi decidió desconocer la regla según la cual el presidente de la Corte debía ser su miembro más antiguo en tanto que este fue uno de los jueces que votó a favor de la doctrina de la estructura básica ${ }^{58}$. En 1975 una corte regional anuló la reelección de GANDHI porque, al parecer, había cometido actos de corrupción. Como consecuencia de esta decisión, GANDHI reaccionó declarando un estado de emergencia en el que se restringió fuertemente la libertad de prensa y se privó de la libertad a varios opositores ${ }^{59}$. Además de eso, propuso una serie de enmiendas a la Constitución: en menos de una semana, gracias a sus mayorías parlamentarias, se aprobaron tres reformas que buscaban dejar sin efecto la doctrina de la estructura básica y que excluían de cualquier clase de control judicial la elección del Primer Ministro $^{60}$. Nuevamente con muchas presiones por parte del ejecutivo, la Corte decidió que la elección de Gandhi era válida, pero declaró la nulidad de la enmienda que impedía cualquier control judicial sobre las elecciones por quebrantar elementos de la estructura básica de la Constitución ${ }^{61}$.

Como resultado, GANDHI promovería una nueva enmienda que, entre otros, señalaba que el control judicial con fundamento en la estructura básica estaba proscrito (enmienda 42-secc. 55). En 1980 la lucha por el dominio interpretativo de la Constitución se cerraría con el fallo Minerva Mills v.

56 Kesavananda Bharati v. State of Kerala. Corte Suprema de Justicia de la India, 1973. Fecha de consulta: 27 de mayo de 2015. Disponible en: http://judis.nic.in/supremecourt/imgs1. aspx?filename $=29981$

57 Cfr. Neuborne, ob. cit., 492 y Prateek, S. Today's Promise, Tomorrow's Constitution: 'Basic Structure', Constitutional Transformations and the Future of Political Progress in India. National University of Juridical Sciences, 2008, 1(3), 417.

58 Mate, M. Two Paths to Judicial Power: The Basic Structure Doctrine and Public Interest Litigation in Comparative Perspective. San Diego International Law Journal, 2010-2011, 12 , 185 y Templeman, L. The Supreme Court and the Constitution. En Kirpai et al., eds., Supreme but not Infallible, cit., 52.

59 Una descripción del estado de emergencia decretado puede verse en NeuBorne, ob. cit., 492 y Krishnamurthy, V. Colonial Cousins: Explaining India and Canada's Unwritten Constitutional Principles. The Yale Journal of International Law, 2009, 34(1), 228.

60 Cfr. SATHE, Judicial activism in India..., cit., 75.

61 Véase el caso Indira Gandhi v. Raj Narain. Corte Suprema de Justicia de la India, 1975. Fecha de consulta: 27 de mayo de 2015. Disponible en: http://judis.nic.in/supremecourt/imgst. aspx?filename $=21564$ 
Union of India ${ }^{62}$, en el cual la Corte declaró inconstitucional dicha reforma afirmando que el control judicial de enmiendas por violación de la estructura básica hacía parte de dicha estructura básica. Lo que iniciaría como una débil doctrina en 1973, se convertiría después, por voto unánime de los magistrados, en un elemento de la misma estructura básica ${ }^{63}$. A partir de entonces cesaron los ataques contra la Corte puesto que ningún otro partido pudo controlar mayoritariamente el Parlamento ${ }^{64}$; la Corte fue considerada por la sociedad como un órgano que salvó la democracia india de la instauración de una dictadura constitucional ${ }^{65}$ y GANDHI perdió el poder por los abusos cometidos durante la emergencia ${ }^{66}$.

\section{LA DERROTA JUDICIAL: VENEZUELA Y HUNGRÍA}

A partir de las anteriores experiencias, a primera vista podría pensarse que la existencia de cortes que desarrollen judicial review de enmiendas sería suficiente para impedir la consolidación de regímenes abusivos. Sin embargo, esto no es suficiente, como pasa a verse con los casos de Venezuela y Hungría.

\subsection{Venezuela}

La elección de Hugo CHÁvEZ como presidente en 1999 tendría una incidencia constitucional significativa en Venezuela. En efecto, al prestar el juramento correspondiente para su posesión, señaló que juraba ante una Constitución moribunda ${ }^{67}$-la de 1961-, y ese mismo día expidió un decreto en el que se le consultaba al pueblo si estaba de acuerdo con la convocatoria a una asamblea constituyente para que redactara una nueva Constitución ${ }^{68}$. La Corte Suprema de Justicia (que en ese entonces realizaba el control de constitucionalidad) profirió varias sentencias en las cuales analizó la constitucionalidad del decreto de convocatoria a la asamblea. En algunos casos consideró que la

62 Minerva Mills v. Union of India. Corte Suprema de Justicia de la India, 1980. Fecha de consulta: 27 de mayo de 2015. Disponible en: http://judis.nic.in/supremecourt/imgs1. aspx ?filename $=4488$

63 Cfr. Ramachandran, R. The Supreme Court and the Basic Structure Doctrine. En KIRPAI et al., eds., Supreme but not Infallible, cit., 119.

64 Véase RaJeEv-Gowda y SRIDHARAN, ob. cit., 3 y 12.

65 Cfr. Datar, A. The Case that Saved Indian Democracy. En: The Hindu, 2013. Fecha de consulta: 27 de mayo de 2015. Disponible en: http://www.thehindu.com/opinion/op-ed/thecase-that-saved-indian-democracy/article4647800.ece

66 Cfr. Robinson, ob. cit., 32.

67 Sobre este punto, véase Segura y Bejarano, ob. cit., 225 y Pizzolo, C. Populismo y rupturas constitucionales: los casos de Venezuela, Bolivia y Ecuador. Estudios Constitucionales, 2007, 5(1), 378 .

68 Algunas particularidades de la Asamblea pueden verse en Rondón, H. Análisis de la Constitución Venezolana de 1999. Caracas: Ex-Libris, 2004, 6. 
asamblea tenía límites pero, en último término, ante la inmensa popularidad de ChÁvez y el consecuente riesgo de que sus decisiones fueran desconocidas, concluyó que dicho cuerpo era soberano e ilimitado ${ }^{69}$.

Luego de que la ciudadanía apoyó la idea de una asamblea nacional constituyente, se eligieron sus integrantes. A pesar de que los movimientos afines al chavismo obtuvieron el 53\% de los votos, debido a unas reglas electorales creadas bajo el auspicio del nuevo presidente, el 96\% de los escaños (123 de 131) fueron ocupados por personas ideológicamente alineadas con $\mathrm{CHÁVEZ}^{70}$. Como resultado, la oposición no tuvo la posibilidad de incidir de manera decisiva en la redacción de la Constitución de 1999 ${ }^{[71]}$. Como observan Corrales y Penfold ${ }^{72}$, se trata de la Constitución más presidencialista de la región porque aumentó de manera perceptible los poderes del Presidente. Así, entre otras medidas, su periodo se extendió de cinco a seis años y se permitió la reelección presidencial por una única oportunidad. Adicionalmente, dada la impopularidad y los crecientes rumores de corrupción que rodeaban a la Corte Suprema, esta fue sustituida por el Tribunal Supremo de Justicia ${ }^{73}$.

Durante los primeros años del gobierno de CHÁvez no hubo ningún intento legal o constitucional directo dirigido a intervenir el Tribunal. No obstante, mediante estrategias de intimidación indirectas -como las manifestaciones multitudinarias que se convocaban antes de que el Tribunal decidiera, para demostrar el apoyo popular a las políticas chavistas o la amenaza del gobierno de que no acataría las decisiones judiciales adversas- se erosionó la independencia judicial ${ }^{74}$.

En el año 2002 hubo un intento de golpe de Estado que duró menos de 48 horas porque CHÁvEZ regresó a su cargo ${ }^{75}$. Este hecho es particularmente relevante porque el Tribunal Supremo decidió no juzgar a los altos oficiales envueltos en dicho intento, lo cual generó perplejidad en el ejecutivo teniendo en cuenta que todos sus miembros habían sido nombrados por una asamblea nacional mayoritariamente gobiernista. Por lo anterior hubo masivas manifestaciones chavistas en contra de la decisión del Tribunal, y la Asamblea

69 Cfr. TAYLOR M. The Limits of Judicial Independence: A Model with Illustration from Venezuela under Chávez. Journal of Latin American Studies, 2014, 46(2), 250 y LANDAU, D. Constitution-Making gone wrong. Alabama Law Review, 2013, 64(5), 943-948.

70 Cfr. Penfold, M.; Corrales, J. y Hernández, G. Los Invencibles: La reelección presidencial y los cambios constitucionales en América Latina. Revista de Ciencia Política, 2014, 34(3), 545 y Corrales, J. y Penfold, M. Venezuela: Crowding out the Opposition. Journal of Democracy, 2007, 18(2), 101.

71 Sobre la falta de representación de la oposición en el proceso de redacción constitucional se puede véase Landau, Constitution-Making gone..., cit., 942 y Segura y Bejarano, ob. cit., 225.

72 Ob.cit., 101.

73 Cfr. TAYLOR, ob. cit., 249 y LANDAU, Constitution-Making gone..., cit., 949.

74 TAYLOR, ob. cit., 249.

75 Sobre el punto Corrales y Penfold, ob. cit., 102. 
Nacional (el nuevo legislativo venezolano) recomendó la remoción de uno de sus integrantes y destituyó de su cargo al autor de la decisión adversa señalada ${ }^{76}$. Dos años después, la oposición presentó una solicitud de revocatoria del mandato del Presidente y la Sala Electoral del Tribunal Supremo avaló dicha petición. Como consecuencia de ello, varios jueces de esta Sala fueron investigados ${ }^{77}$.

Como se observa, el Tribunal no era controlado aún por CHÁvez, por lo cual a estas interferencias informales a su independencia se añadieron otras intervenciones formales o jurídicas ${ }^{78}$ : en 2004 se expidió la Ley Orgánica del Tribunal que aumentó el número de sus integrantes de 20 a 32. Adicionalmente, ante la mencionada destitución del autor de decisiones adversas y la renuncia de dos jueces de la Sala Electoral a causa de las investigaciones, el Presidente nombró 15 jueces leales a su gobierno ${ }^{79}$, con lo cual sometió por completo al Tribunal que, a su vez, aprovechó para dejar sin efectos sus propias decisiones anteriores desfavorables al chavismo ${ }^{80}$. Con este control absoluto sobre el judicial review el presidente presentó en el año 2007 una reforma constitucional para permitir, entre otros, una segunda reelección presidencial ${ }^{81}$ que, a su turno, fue avalada por el Tribunal Supremo ${ }^{82}$. Sin embargo, esta reforma fue rechazada por el electorado en las urnas.

Dos años más tarde, una nueva enmienda sería promovida para permitir la reelección presidencial indefinida. A pesar de que la Constitución dispone que uno de los principios inderogables de la misma es la alternancia en el poder ${ }^{83}$, el Tribunal concluyó que esta enmienda era plenamente constitucional porque este principio no significa que el poder deba obligatoriamente rotarse entre diferentes partidos, "lo que exige es que el pueblo [...] tenga la posibilidad

76 Los detalles de estas solicitudes pueden verse en TAYLOR, ob. cit., 251-252.

77 Ibíd., 253.

78 Diversos autores coinciden en esta afirmación: CASTALDI, L. Judicial Independence Threatened in Venezuela: the removal of Venezuelan judges and the complications of the Rule of Law reform. Georgetown Journal of International Law, 2006, 37(3), 499; Corrales y Penfold, ob. cit., 104; TAYLOR, ob. cit., 253 y HIRSCHL, ob. cit., 750.

79 Cfr. TAYLOR, ob. cit., 254.

80 Véase CASTALDI, ob. cit., 501.

81 Cfr. Brewer-Carías, A. Hacia la creación de un Estado socialista, centralizado y militarista en Venezuela (2007): análisis de la propuesta presidencial de reforma constitucional. Vniversitas, 2007, 114 y Bozo, A. La Democracia, la Soberanía Popular y el Poder Popular a la luz de la Reforma Constitucional Venezolana de 2007. Frónesis, 2008, 15(1).

82 Véase el texto de Brewer-CARías, A. El Juez Constitucional vs. La Supremacía Constitucional. En: allanbrewercarias .com Fecha de consulta: 30 de enero de 2015. Disponible en: http:// www.allanbrewercarias.com/Content/449725d9-f1cb-474b-8ab2-41efb849fea8/Content/567.pdf

83 Sobre los fundamentos de la inalterabilidad de dicho principio véase BREWER-CARÍAS, A. Génesis y Evolución de la Constitución de 1999. En allanbrewercarias.com. Fecha de consulta: 30 de enero de 2015. Disponible en: http://www.brewercarias.com/Content/449725d9f1 cb-474b-8ab2-41 efb849fea2/Content/I,\% 201,\% 20991.\%20GENESIS\%20Y\% 20 EVOLUCI\%C3\%93N\%20DE\%20LA\%20CONSTITUCI\%C3\%93N\%20DE\%201999.\%20 Monte\%C3\%A1vila\%202009.doc).pdf 
periódica de escoger sus mandatarios" ${ }^{"}$. Esta enmienda fue aprobada por el pueblo mayoritariamente y CHÁVEZ se mantuvo 14 años en el poder, hasta la fecha de su muerte. Algunos autores concluyen que durante su presidencia se consolidó un régimen iliberal por cuanto se desdibujaron por completo el principio de separación de poderes ${ }^{85}$ y los derechos fundamentales, así como los derechos de la oposición se restringieron dramáticamente ${ }^{86}$.

\subsection{Hungría}

Luego de la caída de la Unión Soviética, Hungría reformó de manera profunda la Constitución comunista de 1949 para incluir una serie de aspectos propios de regímenes liberales: una amplia carta de derechos, así como un elenco de instituciones -que fuesen contrapeso del Parlamento (unicameral) y del primer ministro-, dentro de las cuales se destaca de manera preponderante la Corte Constitucional ${ }^{87}$. Esta Corte tenía una amplia jurisdicción (con $a c$ tio popularis), y gracias a esta logró desarrollar de manera admirable, en el concierto europeo, los derechos fundamentales y sociales y, simultáneamente, fue un dique efectivo contra los excesos del legislativo y del ejecutivo ${ }^{88}$. Aunque originalmente la Constitución de 1989 establecía que esta podía ser reformada con el consenso de 2/3 partes del Parlamento, para efectos de evitar que una mayoría circunstancial abusara del poder reforma, a mediados de los noventa se añadió un artículo (24.5) que disponía que la creación de una nueva constitución requería del voto afirmativo de las 4/5 partes del Parlamento $^{89}$. De 1989 a 2010, solo en una ocasión el partido liberal logró una mayoría parlamentaria de $2 / 3$ partes, pero la Constitución no fue reformada de manera sustancial ${ }^{90}$.

84 Tribunal Supremo de Justicia de Venezuela, Sentencia 08-1610 de 2009. Fecha de consulta: 15 de enero de 2016. Disponible en: http://historico.tsj.gob.ve/decisiones/scon/Febrero/53-3209-2009-08-1610.html

85 Cfr. Corrales y Penfold, ob. cit., 99.

86 Este el argumento de LóPEz-MAYA, M. Venezuela: The Political Crisis of Post-Chavismo. Social Justice, 2014, 40(4) y LoPerenA, G. Chavez's Charade Democracy in Venezuela. Harvard International Review, 2003, 35(2).

87 Véase Kovács, K. у Tóth, G. Hungary's Constitutional Transformation. European Constitutional Law Review, 2008, 7, 184 y HalmaI, G. The Reform of Constitutional Law in Hungary after the Transition. Legal Studies, 1998, 18(2), 188.

88 Cfr. Halmai, ob. cit., 196; Scheppele, K. Constitutional Coups and Judicial Review: How Transnational Institutions can Strengthen Peak Courts at Times of Crisis (With Special Reference to Hungary). Transnational Law and Contemporary Problems, 2014, 23(1), 68; Arato, A. Post-Sovereign Constitution-Making in Hungary: after success, partial failure, and now what? South African Journal on Human Rights, 2010,26(1), 32 y BRETTER, Z. Halfway or no way? Politeja, 2014, 28, 10-11.

89 Cfr. Kovács, K. у Тóth, G., ob. cit., 187.

90 Cfr. JAKab, A. y Sonnevend, P. Continuity with deficiencies: The New Basic Law of Hungary. European Constitutional Law Review, 2013, 9, 104. 
¡En las elecciones parlamentarias de 2010 el partido Fidesz (y el aliado partido demócrata cristiano) obtuvo el 53\% de apoyo popular ante el descontento por la crisis económica que muchos atribuían al partido liberal ${ }^{91}$. Sin embargo, debido a las reglas electorales existentes, Fidesz logró hacerse con el $68 \%$ de los escaños, vale decir, más de las $2 / 3$ partes del Parlamento ${ }^{92}$, lo cual fue interpretado por ese partido como una "revolución en las urnas"93. La nueva mayoría eligió a VIKTOR ORBÁN como primer ministro y, bajo su liderazgo, se adelantaron múltiples reformas constitucionales a la Constitución postcomunista de 1989. Una de las primeras enmiendas consistió en la eliminación del antedicho artículo 24.5 que exigía 4/5 partes para la derogatoria de la Constitución ${ }^{94}$. De este modo se abrió el camino para la expedición de una nueva Constitución con la aquiescencia de las $2 / 3$ partes del Parlamento controlado por Fidesz ${ }^{95}$.

Sin embargo, antes de la presentación de una nueva Constitución, Fidesz aprobó una serie de leyes promovidas por ORBÁn que creaban algunos impuestos retroactivos de hasta el $98 \%$ sobre las mesadas pensionales de los trabajadores estatales ${ }^{96}$. En octubre de 2010 la Corte Constitucional declaró la inconstitucionalidad de dicho impuesto por quebrantar la seguridad jurídica ${ }^{97}$, ante lo cual el gobierno de ORBÁN respondió con una enmienda rápidamente aprobada por sus mayorías que reproducía, en buena medida, las leyes declaradas inconstitucionales. Sin embargo, en mayo de 2011 la Corte anularía nuevamente otras medidas impositivas del gobierno, lo que generó una reacción mucho más fuerte porque entonces el Parlamento procedió a reformar la Constitución para limitar la jurisdicción de la Corte: en los casos en los que la deuda interna húngara superase el 50\% del PIB, la Corte podría ejercer el control de constitucionalidad de las leyes relativas a impuestos sólo si estas quebrantaban los derechos a la vida, la dignidad humana, el habeas data, la libertad de pensamiento, conciencia o religión, así como los derechos de la ciudadanía húngara ${ }^{98}$. Para JAKAB y SONNEVEND ${ }^{99}$, esta norma limitante que en apariencia es transitoria resulta ser, en realidad, permanente puesto que la deuda generalmente supera el $80 \%$ del PIB.

91 Véase DeÁk, A. Hungarian Dances-The Origins and the Future of Viktor Orbán's Revolution. Lithuanian Annual Strategic Review, 2013, 11(1), 150.

92 Scheppele, K., ob. cit., 61 y KovÁcs, K. y Tóth, G., ob. cit., 188.

93 El uso se esta expresión es constantemente repetido por los autores para demostrar que Fidesz interpretó esta victoria como una revolución. Véase DEÁK, ob. cit., 145; BRETTER, Z., ob. cit., 6 y RUPNIK, J. Hungary's Illiberal Turn. How things went wrong. Journal of Democracy, 2012, 23(3), 132.

94 Así lo documentan Kovács y Tóth, ob. cit., 188 y Arato, ob. cit., 43.

95 Cfr. SCHEPPELE, ob. cit., 62-63.

96 KovÁcs, K. у Tóth, G., ob. cit., 192.

97 Véase el análisis de ScHEPPELE, K., ob. cit., 73.

98 Cfr. SCHEPPELE, K., ob. cit., 70.

99 JAKAB, A. y SonNEVEnd, P., ob. cit., 123. 
En julio de 2011, varios ciudadanos intentaron que la Corte declarase la inconstitucionalidad de esta reforma, ante lo cual aquella respondió que la Constitución de 1989 no le otorgaba la posibilidad de adelantar el control de reformas constitucionales por razones de fondo o materiales ${ }^{100}$. Esta decisión sellaría la suerte de la Corte: el gobierno planeaba, desde su llegada, la creación de una nueva Constitución, que sería aprobada con el voto de $2 / 3$ partes del Parlamento en un breve lapso ${ }^{101}$ y que entró a regir en enero de $2012^{[102]}$. La nueva Ley Fundamental de Hungría fue redactada sin la oposición y, debido a las mayorías de Fidesz, refleja de una manera fiel el ideario de este partido y de OrBá́ ${ }^{103}$. Dentro de los puntos más sobresalientes de la nueva Constitución debe destacarse que uno de los objetivos centrales era limitar a las instituciones de control y, en especial, a la Corte Constitucional. En efecto:

- Se mantuvo la restricción en la jurisdicción de la Corte Constitucional en relación con las normas relativas a impuestos, de tal modo que estas medidas no podían ser revisadas a la luz de todos los derechos contenidos en la nueva Ley Fundamental (como los derechos sociales) sino solo de cara a ciertas disposiciones ${ }^{104}$.

- Se eliminó la acción popular, esto es, la posibilidad de que cualquier ciudadano pudiera presentar directamente sus reclamos ante la Corte ${ }^{105}$. Así las cosas, únicamente ciertos funcionarios (cercanos a Fidesz) podrían solicitar la actuación de la Corte $^{106}$.

- Se aumentó el número de integrantes de la Corte de 11 a 15, siendo los nuevos magistrados elegidos por Fidesz ${ }^{107}$.

-Se modificó la forma de elección de los miembros de la Corte Constitucional. Ya no se requiere llegar a un consenso con los partidos de oposición para su elección, sino que basta con el voto de $2 / 3$ partes de los integrantes del Parlamento ${ }^{108}$.

- Se aprobó la enmienda 4 a la Ley Fundamental que deja sin efectos varias tesis jurisprudenciales de la Corte relativas, entre otros, a aspectos tales como la prohibición de criminalizar la indigencia o la obligación de

100 Cfr. Scheppele, ob. cit., 74 y Halmai, G. Unconstitutional Constitutional Amendments: Constitutional Courts as Guardians of the Constitution? Constellations, 2012, 19(2), 182 y 194.

101 Cfr. SCHEPPELE, ob. cit., 64.

102 Cfr. JAKAB, y SONNEVEnd, ob. cit., 102.

103 Cfr. Scheppele, K., ob. cit., 64 y J JKAB, y Sonnevend, ob. cit., 103.

$104 \mathrm{Al}$ respecto véase Kovács, K. y Tóth, G., ob. cit., 202.

105 Véase Scheppele, K., cit., 75.

106 Cfr. JAKAB, y SoNNEVEND, ob. cit., 123.

107 SCHEPPELE, ob. cit., 71 y FleCK, Z. et al. (2011). Opinion on the Fundamental Law of Hungary, 2011, 28. Fecha de consulta: 15 de agosto de 2015. Disponible en: http://lapa.princeton. edu/hosteddocs/amicus-to-vc-english-final.pdf

108 Cfr. Scheppele, ob. cit., 71 y KovÁcs, y Tóth, ob. cit., 193. 
que los estudiantes que hubiesen recibido apoyo estatal para su educación permaneciesen en territorio húngaro por un periodo de tiempo ${ }^{109}$.

- Se modificó el modo de elección de varios órganos de control para que Fidesz pudiese elegirlos ${ }^{110}$.

En 2013, la enmienda 4 fue analizada por la Corte Constitucional, la cual concluyó que no tenía competencia para examinar la constitucionalidad de reformas constitucionales. En 2014 Fidesz obtendría una nueva mayoría en el Parlamento y Orbán sería reelegido como Jefe de Gobierno ${ }^{111}$, con lo cual la "constitutional revenge" en contra de la Corte terminó por someterla al ejecutivo ${ }^{112}$.

\section{CONCLUSIONES}

Como refiere SCHePPeLE ${ }^{113}$, a pesar de que se tenía la esperanza de que las cortes creadas en democracias en transición serían guardianas de la democracia constitucional, muchas de ellas han sucumbido ante el poder de otros actores estatales. La pregunta que surge en este punto tiene que ver entonces con cuáles son los factores políticos e institucionales que han permitido o impedido que un Tribunal Constitucional joven perdure y se consolide como institución protectora de la democracia. A la luz de los cuatro casos estudiados, a continuación se ofrecen algunas de esas variables:

- Primero que todo, debe decirse que las cortes de regímenes transicionales no solo son actores jurídicos sino también políticos. En ese sentido, como han señalado varios autores ${ }^{114}$, además de las reglas jurídicas las cortes ponderan elementos extrajurídicos tales como la posibles reacciones provenientes de otras ramas del poder. Existe una probabilidad más alta de que estas reacciones se produzcan en jóvenes democracias, en las cuales las herramientas jurídicas se utilizan para lograr fines autoritarios (i.e., constitucionalismo abusivo). En ese orden de ideas, si el orden jurídico no es un límite para el gobernante (sino más bien un instrumento para ser manipulado a su favor), es necesario estudiar la influencia de los factores extrajurídicos (o políticos) en la labor judicial.

109 Véase Halmai, G. The End of Liberal Constitutionalism in Hungary? En Int'l J. Const. L. Blog, Fecha de consulta: 15 de agosto de 2015. Disponible en: http://www.iconnectblog. com/2013/04/the-end-of-liberal-constitutionalism-in-hungary

110 Esto puede verse en detalle en Bánkuti, M.; Halmai, G. y Scheppele, K. Disabling the Constitution. Journal of Democracy, 2012, 23(3), 140-141, 143-145 y KoRNAI, J. Hungary's U-Turn: Retreating from Democracy. Journal of Democracy, 2015, 26(3), 35.

111 Cfr. KornaI, ob. cit., 42.

112 Cfr. SCHEPPELE, ob. cit., 96 y 106.

113 Véase Scheppele, K. Guardians of the Constitution: Constitutional Court Presidents and the Struggle for the Rule of Law in Post-Soviet Europe. University of Pennsylvania Law Review, 2006, 154(6), 1757-1758.

114 Véase Ginsburg, ob. cit. y a VAROL, ob. cit. 
- En tal contexto, un primer factor fundamental es un respeto mínimo hacia la independencia de las cortes por parte del ejecutivo ${ }^{115}$. Es revelador que en los casos de Colombia e India, si bien existieron fricciones entre las cortes y el ejecutivo, nunca se intentó aumentar el número de jueces para elegir miembros leales al gobierno. Por oposición, una de las muestras más evidentes del desprecio por la existencia de un Tribunal independiente que es considerado como un obstáculo, es el aumento de sus integrantes para nombrar jueces proclives a las políticas gubernamentales (Hungría-Venezuela).

- Un segundo factor crucial es el prestigio de las cortes ${ }^{116}$. En tal sentido, tanto la Corte colombiana como la india gozaban de una gran reputación intelectual y académica dentro sus respectivas sociedades y, por consiguiente, tanto ÁlVARO URIBE como INDIRA GANDHI acataron las decisiones contrarias a sus intereses, como muestra de respeto por una institución con amplia aceptación popular ${ }^{117}$. Por el contrario, en el caso venezolano la pésima imagen institucional de la desaparecida Corte Suprema allanó el terreno para su aniquilación y la creación de un nuevo Tribunal dependiente del gobierno.

- En línea con esto, el respeto por las cortes no sólo debe extenderse entre la ciudadanía para efectos de garantizar su perdurabilidad, sino que también debe permear a las demás ramas del poder. Así, una deferencia mínima hacia la labor de las cortes por parte del ejecutivo, así como el respaldo de otras instituciones de control (como organismos internacionales o, a nivel interno, defensorías u oficinas de derechos humanos) es vital para detener un régimen abusivo ${ }^{118}$ como el de Hungría que, como se vio, logró controlar todas estas instituciones que podrían ser contrapesos eficaces contra ORBÁN.

- Por otro lado, alguna parte de la literatura que ha estudiado cortes en democracias en transición señala que el campo de acción para un Tribunal Constitucional será más amplio si la distribución del poder es más difusa ${ }^{119}$. Así, por ejemplo, los ataques hacia la Corte Suprema india han desaparecido porque desde la era de INDIRA GANDHI ningún otro partido político ha logrado tener una mayoría amplísima del Parlamento. Por el contrario, la concentración de poder en Venezuela y Hungría ha impedido que sus tribunales

115 Cfr. Tushnet, ob. cit., 438.

116 Véase Scheppele, Guardians of the Constitution..., cit., 1760 y Scheppele, "Constitutional Coups and Judicial Review...", cit., 54.

117 Como bien anotan Dixon y Landau, la doctrina [del control material de enmiendas] está lejos de ser una solución perfecta: esta depende del apoyo popular y judicial, así como de la voluntad política (trad. libre): Dixon, R. y LANDAU, D. Transnational constitutionalism and a limited doctrine of unconstitutional constitutional amendments. En: Social Science Research Network, 2015, 2. Fecha de consulta: 15 de octubre de 2015. Disponible en: http://papers.ssrn. com/sol3/papers.cfm?abstract_id=2624838

118 Sobre este punto concuerdan Scheppele, K., Constitutional Coups and Judicial Review..., ob. cit., 54; Issacharoff, Constitutional Courts and Democratic Hedging..., ob. cit., 1011; ARATO, ob. cit., 44 y HirSCHL, ob. cit., 723, 744 y 748.

119 Esa es la sugerente propuesta de GinsBURG, ob. cit., 17-18. 
constitucionales desarrollen una labor más activa para efectos de diluir el abuso constitucional que allí se vive.

- Adicionalmente, se debe señalar que un escenario ideal para efectos de evitar la formación de un régimen constitucional autoritario es diseñar mecanismos que impidan que el gobernante de turno pueda manipular el derecho para conseguir objetivos contrarios al bien común. Así, es interesante notar que en los cuatro casos reseñados, los mecanismos de reforma constitucional fueron medios de los cuales se valió el ejecutivo (en conjunto con el legislativo) para someter a las cortes. Esto es así dado que en las cuatro experiencias el proceso de enmienda constitucional no impone límites al poder de reforma especialmente rígidos $\mathrm{y}$, por consiguiente, no existe un desincentivo para el gobernante que pretende abusar de las reformas. Por el contrario, es interesante notar cómo en Estados Unidos o en Alemania después de la Segunda Guerra Mundial, los gobernantes no han sido proclives al abuso del poder de reforma, dadas las exigentes condiciones procesales en el caso norteamericano ${ }^{120}$ y materiales en la experiencia europea ${ }^{121}$.

- Finalmente, y dentro del enfoque estratégico anunciado, las cortes deben ser muy prudentes, en el entendido de que deben intervenir contra una política mayoritariamente auspiciada por el gobierno y el legislativo si, y sólo si, es para evitar la destrucción del régimen constitucional ${ }^{122}$. Aunque a primera vista podría pensarse que la doctrina de la estructura básica de India es excesivamente desconfiada respecto de las instituciones mayoritarias, debe tenerse en cuenta que esta salvó a este país de la consolidación de una dictadura constitucional. Una Corte agresiva que bloquee sistemáticamente las políticas de un gobierno popular fracasará porque el gobernante percibirá que esta es un opositor político que debe contenerse ${ }^{123}$.

120 Sobre los muy exigentes requisitos formales para reformar la Constitución Federal véase Benítez R., V. F. Constitución popular, no judicial. Una teoría democrática del control de constitucionalidad de las reformas a la Constitución en Colombia. Bogotá: Temis y Universidad de La Sabana, 2014, 59-64. Estas exigencias han llevado a que la Corte Suprema de Justicia norteamericana se abstenga de realizar un control de constitucionalidad a las enmiendas. Véase el caso Coleman v. Miller, 307 U.S. 433 (1939).

121 Sobre la cláusula de eternidad dispuesta en el artículo 79.3 de la Ley Fundamental de Bonn, así como la prudente jurisprudencia del Tribunal Constitucional Federal alemán en relación con las reformas, véase BENítez ob. cit., 113-163.

122 Cfr. Dixon y LANDAu, ob. cit., 627 y YaP, ob. cit., 132.

123 Cfr. IssacharofF, Constitutional Courts and Consolidated..., cit., 611 y GARDBAUM, ob. cit., 303-306. 


\section{BIBLIOGRAFÍA}

Albert, R. Amending Constitutional Amendment Rules. International Journal of Constitutional Law. 2015, 13(3), 655-685.

Arato, A. Post-Sovereign Constitution-Making in Hungary: After Success, Partial Failure, and now what? South African Journal on Human Rights, 2010, 26(1), 19-44.

Austin, G. The Supreme Court and the Struggle for Custody of the Constitution. En B. N. KIRPAI et al., eds. Supreme but not Infallible, Essays in Honour of the Supreme Court of India. Nueva Delhi: Oxford University Press, 2011, 1-15.

Bánkuti, M.; Halmai, G. y Scheppele, K. Disabling the Constitution. Journal of Democracy, 2012, 23(3), 138-146.

Benítez R., V. F. Constitución popular, no judicial. Una teoría democrática del control de constitucionalidad de las reformas a la Constitución en Colombia. Bogotá: Temis y Universidad de La Sabana, 2014.

Bernal-Pulido, C. Transitional Justice within the Framework of a Permanent Constitution: The Case Study of the Legal Framework for Peace in Colombia. Cambridge Journal of International and Comparative Law, 2014, 3(4), 1136-1163.

Bhanu-Menta, P. The Rise of Judicial Sovereignty. Journal of Democracy, 2007, 18(2), 70-83.

Bozo, A. La Democracia, la Soberanía Popular y el Poder Popular a la luz de la Reforma Constitucional Venezolana de 2007. Frónesis, 2008, 15(1), 58-71.

Bretter, Z. Halfway or no way? Politeja, 2014, 28, 5-30.

Brewer-Carías, A. Hacia la creación de un Estado socialista, centralizado y militarista en Venezuela (2007): análisis de la propuesta presidencial de reforma constitucional. Vniversitas, 2007, 114, 145-190.

CAJAs, M. A. El control judicial a la reforma constitucional, Colombia, 1910-2007. Cali: Universidad ICESI, 2008.

CARrillo, F. Constitucionalizar la democracia social. El proceso constituyente de 1991 y América Latina. Bogotá: Temis, 2011.

CAstaldi, L. Judicial Independence Threatened in Venezuela: The removal of Venezuelan judges and the complications of the Rule of Law reform. Georgetown Journal of International Law, 2006, 37(3), 477-506.

Cepeda-Espinosa, M. J. Judicial Activism in a Violent Context: The Origin, Role, and Impact of the Colombian Constitutional Court. Washington University Global Studies Law Review, 2004 3(4), 529-700.

Cepeda-Espinosa, M. J., Transcript: Social and Economic Rights and the Colombian Constitutional Court. Texas Law Review, 2010-2011, 89(7), 1699-1705.

Corrales, J. y Penfold, M. Venezuela: Crowding out the Opposition. Journal of Democracy, 2007, 18(2), 99-113. 
Das, G. The Supreme Court: An Overview. En B. N. Kirpai et al., eds., Supreme but not Infallible, Essays in Honour of the Supreme Court of India. Nueva Delhi: Oxford University Press, 2011, 15-47.

DeÁk, A. Hungarian Dances - The Origins and the Future of Viktor Orbán's Revolution. Lithuanian Annual Strategic Review, 2013, 11(1), 145-168.

Dixon, R. y Landau, D. Transnational constitutionalism and a limited doctrine of unconstitutional constitutional amendments. International Journal of Constitutional Law, 2015, 13(3), 606-638.

Dugas, J. The Origin, Impact and Demise of the 1989-1990 Colombian Student Movement: Insights from Social Movement Theory. Journal of Latin American Studies, 2011, 33(4), 807-837.

Gardbaum, S. Are Strong Constitutional Courts Always a Good Thing for New Democracies? Columbia Journal of Transnational Law, 2014-2015, 53(2), 285-320.

Ginsburg, T. Judicial Review in New Democracies. Nueva York: Cambridge University Press, 2006.

Halmai, G. The reform of Constitutional Law in Hungary after the transition. Legal Studies, 1998, 18(2), 188-196.

Halmai, G. Unconstitutional Constitutional Amendments: Constitutional Courts as Guardians of the Constitution? Constellations, 2012, 19(2), 182-203.

Hirschl, R. The New Constitutionalism and the Judicialization of Pure Politics Worldwide. Fordham Law Review, 2006, 75(2), 721-754.

Issacharoff, S. Constitutionalizing Democracy in Fractured Societies. Texas Law Review, 2003-2004, 82(7), 1861-1893.

Issacharoff, S. Constitutional Courts and Democratic Hedging. Georgetown Law Journal, 2010-2011, 99(4), 961-1012.

Issacharoff, S. Constitutional Courts and Consolidated Power. The American Journal of Comparative Law, 2014, 62(3), 585-612.

Jakab, A. y Sonnevend, P. Continuity with deficiencies: The New Basic Law of Hungary. European Constitutional Law Review, 2013, 9, 104-138.

KornaI, J. Hungary's U-Turn: Retreating from Democracy. Journal of Democracy, 2015, 26(3), 34-48.

Kovács, K. y Tóth, G. Hungary's Constitutional Transformation. European Constitutional Law Review, 2008, 7, 183-203.

Krishnamurthy, V. Colonial Cousins: Explaining India and Canada's Unwritten Constitutional Principles. The Yale Journal of International Law, 2009, 34(1), 207-239.

Landau, D. Abusive Constitutionalism. U. C. Davis Law Review, 2013, 47(1), 189-260.

LANDAU, D. Constitution-Making gone wrong. Alabama Law Review, 2013, 64(5), 923-980. 
Landau, D. A Dynamic Theory of Judicial Role. Boston College Law Review, 2014, 55(5), 1501-1561.

Loperena, G. Chavez's Charade Democracy in Venezuela. Harvard International Review, 2003, 35(2), 13-14.

López-Maya, M. Venezuela: The Political Crisis of Post-Chavismo. Social Justice, 2014, 40(4), 68-87.

Mate, M. Two Paths to Judicial Power: The Basic Structure Doctrine and Public Interest Litigation in Comparative Perspective. San Diego International Law Journal, 2010$2011,12,175-222$.

Moustafa, T. y Ginsburg, T. Introduction: The Function of Courts in Authoritarian Politics. En T. Ginsburg y T. Moustafa, eds. Rule by Law: The Politics of Courts in Authoritarian Regimes. Nueva York: Cambridge University Press, 2010, 1-22.

Neuborne, B. The Supreme Court of India. International Journal of Constitutional Law, 2003, 1(3), 476-510

Osuna, N. La sentencia del referendo: guarda de la Constitución ante el uso instrumental de la democracia. En E. Montealegre, coord., Anuario de Derecho Constitucional: análisis de jurisprudencia de la Corte Constitucional, período 2002 y primer semestre 2003. Bogotá: Universidad Externado de Colombia, 23-40.

Penfold, M.; Corrales, J. y Hernández, G. Los Invencibles: La reelección presidencial y los cambios constitucionales en América Latina. Revista de Ciencia Política, 2014, 34(3), 537-559.

Pizzolo, C. Populismo y rupturas constitucionales: los casos de Venezuela, Bolivia y Ecuador. Estudios Constitucionales, 2007, 5(1), 371-394.

Posada-Carbó, E. Colombia after Uribe. Journal of Democracy, 2011, 22(1), 137-151.

Prateek, S. Today's promise, tomorrow's Constitution: 'Basic Structure', constitutional transformations and the future of political progress in India. National University of Juridical Sciences, 2008, 1(3), 417-498.

Quinche, M. F. Derecho constitucional colombiano. De la Carta de 1991 y sus reformas. Bogotá: Temis, 2012.

Rajeev-Gowda, M. V. y Sridharan, E. Parties and the Party System, 1947-2006. En S. Ganguly et al., eds., The State of India's Democracy. Baltimore: The Johns Hopkins University Press, 2007, 3-25.

Ramachandran, R. The Supreme Court and the Basic Structure Doctrine. En B. N. Kirpai et al., eds., Supreme but not Infallible, Essays in Honour of the Supreme Court of India. Nueva Delhi: Oxford University Press, 2011, 107-133.

Ramírez Cleves, G. El control material de las reformas constitucionales mediante acto legislativo a partir de la jurisprudencia establecida en la Sentencia C-551 de 2003. Revista Derecho del Estado. 2006, 18, 3-32. 
Revelo, J. La Defensoría del Pueblo: el silencio complaciente. En M. García y J. Revelo, eds., Mayorías sin Democracia. Desequilibrio de poderes y Estado de Derecho en Colombia, 2002-2009. Bogotá: DeJusticia, 2009, 146-175.

Robinson, N. Expanding Judiciaries: India and the rise of the Good Governance Court, Washington University Global Studies Law Review, 8(1), 2009, 1-69.

Rondón, H. Análisis de la Constitución Venezolana de 1999. Caracas: Ex-Libris, 2004.

RoznaI, Y. Unconstitutional Constitutional Amendments - The Migration and Success of a Constitutional Idea. American Journal of Comparative Law, 2013, 61(3), 657-719.

Rubiano, S. La Corte Constitucional: entre la independencia judicial y la captura política. En M. García y J. Revelo, eds., Mayorías sin Democracia. Desequilibrio de poderes y Estado de Derecho en Colombia, 2002-2009. Bogotá: DeJusticia, 2009, 84-145.

Rubiano, S. La independencia del Banco de la República y la reelección presidencial. En M. García y J. Revelo, eds., Mayorías sin Democracia. Desequilibrio de poderes y Estado de Derecho en Colombia, 2002-2009. Bogotá: DeJusticia, 2009, 207-243.

RuPNIK, J. Hungary's Illiberal Turn. How things went wrong. Journal of Democracy, 2012, 23(3), 132-137.

SAthe S.P. India: From Positivism to Structuralism. En J. Goldsworthy, ed., Interpreting Constitutions. A comparative study. Oxford: Oxford University Press, 2009, 215-265.

SATHE S.P. Judicial activism in India.Transgressing Borders and Enforcing Limits. Nueva Delhi: Oxford University Press, 2011.

Scheppele, K. Guardians of the Constitution: Constitutional Court Presidents and the Struggle for the Rule of Law in Post-Soviet Europe. University of Pennsylvania Law Review, 2006, 154(6), 1756-1851.

Scheppele, K. Constitutional Coups and Judicial Review: How Transnational Institutions can Strengthen Peak Courts at Times of Crisis (With Special Reference to Hungary). Transnational Law and Contemporary Problems, 2014, 23(1), 51-117.

Segura, R. y Bejarano, A. ¡Ni una asamblea más sin nosotros! Exclusion, Inclusion, and the Politics of Constitution-Making in the Andes. Constellations, 2004, 11(2), 217-236.

TAYLOR, M. The Limits of Judicial Independence: A Model with Illustration from Venezuela under Chávez. Journal of Latin American Studies, 2014, 46(2), 229-259.

Templeman, L. The Supreme Court and the Constitution. En B. N. Kirpai et al., eds., Supreme but not Infallible, Essays in Honour of the Supreme Court of India. Nueva Delhi: Oxford University Press, 2011, 48-56.

Tushnet, M. Authoritarian Constitutionalism. Cornell Law Review, 2014-2015, 100(2), 391-461.

Uprimny, R. The Recent Transformation of Constitutional Law in Latin America: Trends and Challenges. Texas Law Review, 2011, 89(7), 1587-1609. 
Valencia-Villa, H. Cartas de batalla. Una crítica del constitucionalismo colombiano. Bogotá: Panamericana, 2010.

VArol, O. Stealth Authoritarianism. Iowa Law Review, 2015, 100 (4), 1673-1742.

YAP, P. J. The conundrum of unconstitutional constitutional amendments. Global Constitutionalism, 2015, 4(1), 114-136.

\section{Recursos de Internet}

Brewer-Carías, A. El Juez Constitucional vs. La Supremacía Constitucional, 1-46. En: allanbrewercarias.com. Fecha de consulta: 30 de enero de 2015. Disponible en: http:// www.allanbrewercarias.com/Content/449725d9-f1cb-474b-8ab2-41efb849fea8/Content/567.pdf

BREwer-CARías, A. Génesis y evolución de la Constitución de 1999, 1-20. En: allanbrewercarias. com. Fecha de consulta: 30 de enero de 2015. Disponible en: http://www.brewercarias com/Content/449725d9-f1cb-474b-8ab2-41efb849fea2/Content/I,\%201,\%20991.\%20GENESIS\%20Y\%20EVOLUCI\%C3\%93N\%20DE\%20LA\%20CONSTITUCI\%C3\%93N\%20 DE\%201999.\%20Monte\%C3\%A1vila\%202009.doc).pdf

Dixon, R. y Landau, D. Transnational constitutionalism and a limited doctrine of unconstitutional constitutional amendments. En: Social Science Research Network, 2015. Fecha de consulta: 15 de octubre de 2015. Recuperado de: http://papers.ssrn.com/sol3/ papers.cfm?abstract_id=2624838

Datar, A. The case that saved Indian democracy. En: The Hindu, 2013. Fecha de consulta: 27 de mayo de 2015. Disponible en: http://www.thehindu.com/opinion/op-ed/the-casethat-saved-indian-democracy/article4647800.ece

Fleck, Z. et al. (2011). Opinion on the Fundamental Law of Hungary, 2011, 1-40. Fecha de consulta: 15 de agosto de 2015. Disponible en: http://lapa.princeton.edu/hosteddocs/ amicus-to-vc-english-final.pdf

Golaknath v. State of Punjab. Corte Suprema de Justicia de la India, 1967. Fecha de consulta: 27 de mayo de 2015. Disponible en: http://judis.nic.in/supremecourt/imgs1. aspx?filename $=2449$

Halmai, G. The End of Liberal Constitutionalism in Hungary? En Int'l J. Const. L. Blog, Fecha de consulta: 15 de agosto de 2015. Disponible en: http://www.iconnectblog. com/2013/04/the-end-of-liberal-constitutionalism-in-hungary

Indira Gandhi v. Raj Narain. Corte Suprema de Justicia de la India, 1975. Fecha de consulta: 27 de mayo de 2015. Disponible en: http://judis.nic.in/supremecourt/imgst. aspx?filename $=21564$

Kesavananda Bharati v. State of Kerala. Corte Suprema de Justicia de la India, 1973. Fecha de consulta: 27 de mayo de 2015. Disponible en: http://judis.nic.in/supremecourt/ imgs1 .aspx? filename $=29981$

Minerva Mills $v$. Union of India. Corte Suprema de Justicia de la India, 1980. Fecha de consulta: 27 de mayo de 2015. Disponible en: http://judis.nic.in/supremecourt/imgs1. aspx?filename $=4488$ 
Sankari Prasad v. Union of India. Corte Suprema de Justicia de la India, 1951. Fecha de consulta: 14 de enero de 2014. Disponible en http://judis.nic.in/supremecourt/imgs1. aspx?filename $=1174$

Sentencia 08-1610 de 2009. Tribunal Supremo de Justicia de Venezuela, 2009. Fecha de consulta: 27 de mayo de 2015. Disponible en: http://historico.tsj.gob.ve/decisiones/ scon/Febrero/53-3209-2009-08-1610.html

Sentencia C-141 de 2010. Corte Constitucional de Colombia, 2010. Fecha de consulta: 27 de mayo de 2015. Disponible en: http://www.corteconstitucional.gov.co/ relatoria/2010/c-141-10.htm

Sentencia C-574 de 2011. Corte Constitucional de Colombia, 2011. Fecha de consulta: 27 de mayo de 2015. Disponible en: http://www.corteconstitucional.gov.co/ relatoria/2011/c-574-11.htm

Sentencia C-1040 de 2005. Corte Constitucional de Colombia, 2005. Fecha de consulta: 27 de mayo de 2015. Disponible en: http://www.corteconstitucional.gov.co/ relatoria/2005/C-1040-05.htm 